\title{
PRESENCIA DE PESTICIDAS EN LA DIETA COMO FACTOR DE RIESGO PARA EL PARKINSON ESPORÁDICO
}

\section{${ }^{1}$ Sequeira Quesada, Carlos Mario y ${ }^{2}$ Arias Rivera, Daniela}

${ }^{1}$ Estudiante de Bachillerato en Ciencias Médicas y Licenciatura en Medicina y Cirugía, Universidad de Costa Rica, San José, Costa Rica.

${ }^{2}$ Estudiante de Bachillerato y Licenciatura en Nutrición, Universidad de Costa Rica, San José, Costa Rica.

Resumen: El Parkinson es una de las enfermedades neurodegenerativas más comunes a nivel mundial, cuyas causas específicas no se han podido esclarecer completamente; de hecho, en la actualidad se entiende esta enfermedad como un producto de la interacción entre factores ambientales, la exposición a pesticidas, factores genéticos y envejecimiento. En la presente investigación se pretenden revisar las bases y principios conocidos de la enfermedad del Parkinson, explicar mecanismos de degeneración neuronal y puntualizar en pesticidas específicos con los cuales se haya comprobado causalidad. Además, se abordará la presencia de dichas toxinas en alimentos de ingesta rutinaria y el posible riesgo que esto propone, lo anterior resultado de una búsqueda bibliográfica. Se encontró que el riesgo de padecer la enfermedad de Parkinson aumenta con la exposición laboral o dietaria a pesticidas organofosforados y organoclorados. Se concluye que la incorrecta utilización de pesticidas puede generar contaminación en aguas, suelos y los alimentos donde se utilicen, incrementando el riesgo de padecer la enfermedad de Parkinson.

Palabras clave: Parkinson, organoclorados, organofosforados, neurodegeneración 


\title{
PRESENCE OF PESTICIDES IN DIET AS A RISK FACTOR FOR SPORADIC PARKINSON'S DISEASE
}

\begin{abstract}
Parkinson is one of the most common neurodegenerative diseases worldwide. Nevertheless specific causes are up today not elucidated completely; as a matter of fact, this disease is understood nowadays as a result of the interaction between environmental influences such as exposure to pesticides, heritage and aging. In this research it is intended to review the bases and known principles of Parkinson's disease, to explain some of the mechanisms for neuronal degeneration and identify specific pesticides that have proven causality between their use and Parkinson's. It will also address the presence of those toxins in food of habitual intake and the risk it may hold, all of this as a result of a bibliographic research. The risk of having Parkinson's disease was found to be greater when occupational-related or dietary exposure of organophosphate and organochlorine pesticides. It is concluded that an incorrect use of pesticides can produce water, soil and food contamination increasing the risk of suffering Parkinson's disease.
\end{abstract}

Key words: Parkinson, organochlorines, organophosphates, neuronal degeneration

\section{INTRODUCCIÓN}

El Parkinson, es la segunda enfermedad neurodegenerativa progresiva más común, superada en incidencia únicamente por el Alzheimer [1,2], con una prevalencia de un $1 \%$ en la población de adultos mayores que superan los 60 años y que se quintuplica al alcanzar los 85 años de edad [1]. Se caracteriza por una pérdida de las neuronas dopaminérgicas de la sustancia nigra pars compacta, y su sintomatología típica es sólo evidente al alcanzarse depleciones del 50 a $70 \%$ [2]. Va acompañada con la aparición de inclusiones celulares de una apariencia histológica eosinófila, así como de dendritas distróficas anormales, ambas formadas mayoritariamente por la acumulación de proteínas [1,3,4].

Los signos y síntomas motores característicos son: temblor en reposo, rigidez (hipertonía), dificultad para iniciar movimientos (aquinesia), lentitud al realizar los movimientos (bradiquinesia) y disminución en la amplitud de movimientos (hipoquinesia) [5], además de otros menos comunes como la camptocormia (flexión severa del tronco al estar en bipedestación o sentado) [6], balanceo de brazos disminuido, Revista electrónica publicada por el Departamento de Farmacología de la Escuela de Medicina de la Universidad de Costa Rica, 2060 San José, Costa Rica. ${ }^{\circledR}$ All rights reserved. Licensed under a Creative Commons Unported License. reducción de expresividad facial y volumen de voz, aceleración en segmentos del discurso y fatiga [7].

También hay presencia de signos y síntomas no motores que conllevan una vital importancia al desafiar el estilo de vida rutinario de los enfermos: demencia, problemas para dormir, psicosis $\mathrm{y}$ disfunción autonómica, aunque se debe hacer la separación entre las condiciones propias del Parkinson y de aquellas propiciadas por el tratamiento (iatrogénicas) [8].

A pesar de múltiples esfuerzos y estudios, la patogénesis y etiología del Parkinson no se han logrado dilucidar completamente, aunque hoy se entiende como un resultado de la interacción entre factores ambientales, genéticos y del envejecimiento [9]. El Parkinson producido por mutaciones genéticas no es tan común y ocupa tan solo un $10 \%$ del total de casos [10,1]. Esta información, reforzada por estudios con gemelos monocigóticos y dicigóticos confirma que la mayoría de casos de Parkinson son esporádicos, no hereditarios, sin una base genética definida y $\sin$ un origen conocido $[2,10,11]$. 
A raíz de estos hallazgos el estudio de factores ambientales como catalizadores del Parkinson ha tomado vigor. En 1982 el interés en estas investigaciones se intensificó al notar que la sustancia 1-metil-1,2,3,6-tetrahidropiridina (MPTP) reproducía casi en su totalidad los signos y síntomas del Parkinson [11]. Esto constituyó la primera prueba que una toxina ambiental podía ser causante de esta condición [10]. Se ha pensado en pesticidas actuando como esta neurotoxina [12]. Estudios han identificado a diferentes pesticidas (insecticidas y herbicidas), como posibles precursores del Parkinson [13,14], e inclusive ha sido posible la dilucidación de probables mecanismos por los cuales estos ejercen su efecto nocivo sobre las neuronas dopaminérgicas de la sustancia nigra pars compacta [9].

El conocimiento de que los pesticidas degeneran estas neuronas ha sido utilizado inclusive en modelos animales para el estudio de la condición parkinsoniana [10]. La literatura y diversos estudios confirman la relación entre exposición a pesticidas y una mayor incidencia de Parkinson, además de encontrar niveles elevados en suero y tejido cerebral de determinados pesticidas al realizar autopsias y necropsias de pacientes que sufrieron esta enfermedad $[12,13,15]$. La mayoría de la población no vive en un ambiente rural, por lo que no peligra en tan grave medida de exposición ocupacional, la cual aumenta la incidencia del Parkinson[16]. Es por esto que se requiere determinar otras maneras por las cuales se puedan exponer a estas sustancias. Figuran entre ellas la alimentación por el alto uso de pesticidas en la agroindustria, pues éstos son considerados el factor principal de la producción agrícola y de la calidad de la cosecha [17].

Este artículo tiene como objetivo revisar las bases y principios conocidos de la enfermedad del Parkinson, explicar mecanismos de degeneración neuronal y puntualizar en pesticidas específicos con los cuales se haya comprobado causalidad, así como abordar la presencia de dichas toxinas en alimentos de ingesta rutinaria y el posible riesgo que esto conlleva.

\section{GANGLIOS BASALES EN EL CONTROL MOTOR}

Los ganglios basales son el conjunto de agrupaciones de sustancia gris adentrados en la sustancia blanca de los hemisferios cerebrales [5]. Consisten del caudado, el putamen y el globo pálido [18]. El caudado y el putamen forman el cuerpo estriado, llamado así por el aspecto que producen las líneas de sustancia gris al atravesar la cápsula interna [5]. El núcleo subtalámico y la sustancia nigra tienen una muy estrecha relación con los ganglios basales, sin embargo estos no deben ser considerados propiamente como tales $[5,18]$. La sustancia nigra se puede separar en dos, una parte compacta y otra reticulada, según el aspecto que le concedan la disposición de las células en ellas, además de las aferencias y eferencias [18].

Los ganglios basales están dispuestos topográfica y funcionalmente en un circuito cortico-estriatopálido-tálamo-cortical (hipótesis del circuito segregado) [19] por medio del cual construyen un patrón de movimiento, integrando información ambiental, deseos y experiencia, es decir, controlan la intención de realizar un movimiento [20]. El primer modelo de esta arquitectura fue propuesto en 1980[21].

El circuito inicia con las fibras corticoestriadas, que comunican la corteza cerebral con el cuerpo estriado [5), con la notable excepción de la corteza auditiva primaria y la corteza visual primaria $[5,18]$. Estas fibras harán sinapsis con las neuronas espinosas medianas que se encuentran en el cuerpo estriado. La naturaleza de las señales que viajan por esta vía no está completamente dilucidada, sin embargo el caudado recibirá información de cortezas asociativas frontales mientras que el putamen recibe aferencias de las cortezas motoras y premotoras [22].

Las neuronas espinosas medianas son GABAérgicas (inhibitorias) y se encuentran dispuestas en compartimientos dentro del cuerpo estriado: los estriosomas y la matriz, siguiendo la organización en capas que poseen el resto de neuronas

Revista electrónica publicada por el Departamento de Farmacología de la Escuela de Medicina de la Universidad de Costa Rica, 2060 San José, Costa Rica. ${ }^{\circledR}$ All rights reserved. Licensed under a Creative Commons Unported License. 
importantes en estructuras del teléncefalo [20,23]. Las neuronas espinosas medianas también se pueden diferenciar según sus eferencias $y$ neuromoduladores usados. Un grupo se proyectará hacia el globo pálido interno en la llamada vía directa mientras que otra agrupación de neuronas conformará la vía indirecta comunicándose con el globo pálido externo [20,22]. Las neuronas, en ambos globos pálidos, con las cuales las neuronas espinosas medianas hacen sinapsis son de naturaleza GABAérgica [18].

En la vía directa, la corteza mediante sinapsis glutamatérgicas (excitatorias) estimula las neuronas medianas espinosas. Estas se proyectan a la sustancia nigra reticular y el globo pálido interno [20] inhibiendo transitoriamente este último. El globo pálido interno se proyecta hacia el tálamo, a sus núcleos ventral lateral y anterior [22], donde ejerce regularmente una inhibición tónica [18]. Las fibras excitatorias talamocorticales viajarán de estos núcleos a las cortezas motoras y premotoras [22]. Al darse la inhibición del globo pálido interno, se elimina la inhibición tónica que este ejercía, provocando la activación talámica de las cortezas motoras y premotoras y así desencadenando todos los movimientos posibles [22]. En la vía indirecta, las neuronas espinosas medianas envían sus eferencias inhibitorias al globo pálido externo [20] y harán sinapsis en el núcleo subtalámico, al cual inhiben tónicamente. Sin embargo, al estar inhibido el globo pálido externo, el núcleo subtalámico se preserva activo y excita al globo pálido interno, lo que deriva en la inhibición del tálamo [22]. Es decir, el control motor ejercido por los ganglios basales depende del balance entre las vías directas e indirecta, puesto que la primera desinhibe la realización de movimientos, mientras que la segunda actúa como su freno [18].

De gran interés resulta a su vez el circuito nigroestriatal [22], compuesto por las fibras nigroestriadas que viajan de la sustancia nigra compacta hacia el cuerpo estriado y utilizan la dopamina como neurotransmisor [5]. Las neuronas espinosas medianas que pertenecen a la vía directa expresan receptores $\mathrm{D}_{1}$ los cuales aumentan su excitabilidad, mientras que las de la vía indirecta expresan receptores $\mathrm{D}_{2}$ con el efecto contrario [20]. Las neuronas dopaminérgicas mantienen una tasa de disparo constante en condiciones normales y a través de estas conexiones favorecen la desinhibición talámica [24].

Lesiones y degeneraciones de este circuito ocasionan los signos y síntomas motores del Parkinson ya mencionados $[7,22]$. Esta condición se asocia con la degeneración progresiva de la sustancia nigra pars compacta causando disfunción estriatal [25]. La pérdida de estas neuronas dopaminérgicas resulta en una excitación excesiva del núcleo subtalámico y el globo pálido interno [24], porque la reducción de dopamina favorece la activación de las neuronas espinosas medianas que se proyectan al globo pálido externo, mientras que aquellas que comunican con el globo pálido interno ven reducida su excitación [26], resultando en una inhibición mayor de lo normal de las proyecciones talamocorticales y provocando los signos motores ya mencionados [24].

\section{GENERALIDADES DE PESTICIDAS: ORGANOFOSFORADOS $Y$ ORGANOCLORADOS}

Un pesticida es una sustancia química, natural o sintética, utilizada en agroindustria con el fin de controlar organismos perjudiciales; y según su capacidad de contaminar los alimentos se pueden clasificar en: organoclorados y organofosforados [27].

Los pesticidas organoclorados poseen una alta liposolubilidad y resisten fuertemente la degradación, por lo que son extremadamente persistentes y difíciles de eliminar del medio ambiente [13]. ]. Por ejemplo, el lindano, un isómero del hexaclorocicloexano técnico (pesticida organoclorado), era el ingrediente activo de algunos productos para controlar plagas en casas y jardines, en granjas, silvicultura y en la cría de animales; este pesticida fue muy utilizado en Costa Rica antes de año 2000, actualmente se encuentra prohibido su uso [28]. 
Por otro lado, los pesticidas organofosforados son poco persistentes en el ambiente, de 2 a 8 semanas a comparación de los organoclorados que perduran de 300 a 550 semanas, dependiendo del pesticida específico que sea, $y$ se degradan rápidamente en el organismo animal, sin embargo, se debe tener en cuenta que los metabolitos secundarios generados son de difícil evaluación toxicológica [27]. En la actualidad, los pesticidas organofosforados son los que se utilizan mayormente, especialmente luego de que los organoclorados fueran restringidos en su uso debido a preocupaciones sobre bioacumulación y efectos tóxicos [13].

\section{MECANISMOS DE ACCION DE PESTICIDAS Y SU RELACIÓN CON LA ENFERMEDAD DE PARKINSON}

Ambos tipos de pesticidas actúan de diferente manera sobre sus organismos blanco. Según el Ing. Oscar Arias Madriz, quien actualmente labora con la empresa estadounidense ExcelAg, Corp., los pesticidas organoclorados atacan el sistema nervioso central, tanto de la plaga como de mamíferos en general. Específicamente actúan sobre el axón, interfiriendo con el transporte de los iones sodio y potasio, prolongando la apertura de canales de sodio debido a la inhibición de la proteína transportadora $\mathrm{Ca}^{+2}$ ATPasa [29]. Se provoca entonces un estado de sobreexcitación cerebral, manifestándose principalmente por medio de convulsiones; también puede provocarse parestesia, agitación involuntaria, ataxia, hiperreflexia, náusea diarrea, depresión respiratoria y pérdida de la conciencia [28]. Se ha comprobado que los pesticidas organoclorados son neurotóxicos, y que causan estrés oxidativo y daño en el sistema dopaminérgico de cerebros de roedores [13].

Por otro lado, los pesticidas organofosforados poseen su acción tóxica a nivel sináptico, donde se enlaza covalentemente con la enzima acetilcolinesterasa fosforilándola e inhibiendo su actividad [17,30]. Por lo tanto, se produce un exceso de acetilcolina, esto significa un aumento en la actividad colinérgica o estimulación, $\mathrm{y}$ se muestra por ejemplo en: receptores muscarínicos ubicados en corazón y glándulas sudoríparas, receptores nicotínicos ubicados en músculo esquelético, médula adrenal y ganglios autonómicos, y receptores del sistema nervioso [31].

Es importante destacar, que existe evidencia significativa de que la enfermedad de Parkinson está relacionada con la exposición de pesticidas, principalmente insecticidas y herbicidas que sean de tipo organoclorado [14]. Asimismo, la enfermedad se ha asociado con la exposición de pesticidas tanto en ambiente laboral como de uso doméstico, habitar en zonas rurales, consumir agua de pozos y a un elevado nivel de pesticidas organoclorados en el tejido cerebral [32].

\section{MECANISMOS DE DEGENERACIÓN NEURONAL}

Resultado de estudios y modelos animales en casos de Parkinson causado por mutaciones genéticas [10], algunos autores han trabajado en crear hipótesis sobre un posible mecanismo unificado entre el Parkinson esporádico y el monogénico para la neurodegeneración observada. Este se enfoca en proteínas mal plegadas, que luego de un exitoso episodio de acumulación en determinada zona propagan esta tendencia a diferentes partes del encéfalo [4]. Sin embargo, con la observación de que ciertos pesticidas a pesar de diferir en su mecanismo de acción igualmente inducen muerte neuronal en la sustancia nigra [33], convendría más adherirse a otras vías literarias de pensamiento que nos presentan hipótesis multifactoriales no exclusivas para entender la vulnerabilidad selectiva de estas neuronas [34].

Los motivos de neurodegeneración abordada serán falla mitocondrial, estrés oxidativo, estrés del sistema proteosoma-ubiquitina $y$ ampliaremos en la agregación proteica. Estas vías resultan de interés puesto que se ha comprobado a los pesticidas citados en la sección anterior actuando a través de ellas [9]. Todas estas vías 
están relacionadas y no es fácil determinar cuál ocurrió primero, puesto que todas tienen potencial neurotóxico $[9,34]$.

Las acumulaciones proteicas resultan de gran importancia puesto que además de poder inducir muerte celular por sí mismas, pueden ser causadas por otros de los factores citados anteriormente[9], siendo este el motivo por el cual se le ha centrado como gran efector en la búsqueda del mecanismo unificado de acción. Sin embargo, no ha sido posible explicar en su totalidad la relación de causalidad entre la agregación proteica y la degeneración dopaminérgica [4].

En 1919 Konstantin Nikolaevich Tretiakoff (1892-1956) halló agregados proteicos en la sustancia nigra, a los cuales llamó cuerpos de Lewy, en honor a quién había descrito inclusiones similares en otras regiones del cerebro, así como la presencia de estas agrupaciones en procesos de células nerviosas, posteriormente llamadas neuritas de Lewy [4]. La aparición de neuritas de Lewy precede a la de cuerpos de Lewy, y suelen hallarse en el sistema entérico antes de la aparición de síntomas motores [3,4].

A nivel del sistema nervioso central investigadores han trazado seis etapas de avance en la aparición de los cuerpos de Lewy, iniciando en el bulbo olfatorio y núcleo dorsal motor del vago (se piensa que puede ascender retrógradamente desde el abdomen a través del nervio vago) y prosiguiendo al locus coeruleus, substancia nigra, mesocorteza y neocortezas [4]. La presencia de los cuerpos de Lewy en estas distintas ubicaciones a la sustancia nigra se piensa es responsable de la patología no motora asociada con el Parkinson [4,8]. Estos cúmulos proteicos no aparecen espontáneamente en cada lugar, sino que la proteína mal plegada acumulada que conforma los cuerpos de Lewy viaja de una célula a otra. No se ha logrado explicar la génesis de estos cúmulos en el Parkinson esporádico [1,35,36].

Se ha determinado el componente principal de estas inclusiones: la $\alpha$-sinucleína, una proteína nativamente no plegada, soluble y encontrada en neuronas presinápticas y que se ha relacionado con el reciclaje de vesículas presinápticas [1,2,37]. Los monómeros de esta proteína se juntan para formar protofibrillas, las cuales se agrupan formando la hoja plegada $\beta$ (fibrillas), la conformación de mayor frecuencia en los cuerpos de Lewy. Dentro de estas inclusiones eosinófilas también se han encontrado modificaciones de esta proteína: $\alpha$-sinucleína fosforilada y segmentos truncados tanto en su extremo C-terminal como en el N-terminal, los cuales presentan mayor facilidad para acumularse y formar las estructuras ya mencionadas [3,36,38,39].

Se propone que la acumulación de la $\alpha$ sinucleína se da por una ineficiente degradación de esta proteína. Entre las entidades que han sido relacionadas con la eliminación proteica se citan los proteosomas y lisosomas, siendo las enzimas en cuestión algunas como la calpaina I y la catepsina D (enzima lisosómica). Una mutación de estas proteínas (señalando de nuevo la importancia de encontrar el mecanismo unificado), acción enzimática sobre fibrillas de $\alpha$-sinucleína en lugar de los monómeros o alguna otra falla en estas vías ocasiona la aparición exacerbada de segmentos truncados (son productos normales de la acción de estas enzima, pero no en niveles tan elevados como se encuentra en los cuerpos de Lewy), lo cual empeora la acumulación de esta proteína [36,38]. Además otro factor que propicia la acumulación de la $\alpha$-sinucleína es el estrés oxidativo $[1,37]$.

Defectos en el manejo proteosomal de proteínas se ha asociado con el Parkinson [40]. Esta relación se confirma en estudios en los que al inhibir el proteosoma, logran la manifestación de signos parkinsonianos al causar muerte celular, además de análisis post-mortem de tejido cerebral $[1,40,41]$.

Los cuerpos de Lewy favorecen una mayor acumulación de $\alpha$-sinucleína y otras proteínas mal plegadas por otros mecanismos más allá del enzimático y sería mediante inhibición directa del proteosoma [37], lo que se refleja en la presencia dentro de los cuerpos de Lewy de ubiquitina [42]. Esta puede estar conjugada con agregados amorfos

Revista electrónica publicada por el Departamento de Farmacología de la Escuela de Medicina de la Universidad de Costa Rica, 2060 San José, Costa Rica. ${ }^{\circledR}$ All rights reserved. Licensed under a Creative Commons Unported License. 
de proteínas desplegadas, la $\alpha$-sinucleína misma e incluso a proteínas mitocondriales $[39,43,44]$.

\section{Las conformación oligomérica} (protofibrillas) de la $\alpha$-sinucleína es la que disminuye la actividad del proteosoma [1]. Ejerce este efecto ya sea saturando o secuestrando una o más de las chaperonas o ligasas de la ubiquitina requeridas para el buen funcionamiento del proteosoma[41]. Un mal funcionamiento del proteosoma deriva en la acumulación de la $\alpha$ sinucleína misma [43]. A su vez, el estrés oxidativo también impide el buen funcionamiento del proteosoma [45].

Otro catalizador de estas fallas es una disfunción mitocondrial, la cual ha sido conferida con un rol primario en la patogénesis del Parkinson, y algunos autores aseguran que este mal funcionamiento es tan necesario para que se dé la condición como la misma acumulación de proteínas (cuerpos de Lewy) [37, 45, 46].

El vínculo entre mitocondrias y enfermedades de envejecimiento reside en la cadena respiratoria [44]. Autopsias han encontrado una actividad disminuida en la sustancia nigra del complejo I de la cadena respiratoria. Un acontecimiento como este facilita la inducción de la apoptosis por moléculas pro-apoptóticas externas (por ejemplo Bax) [45]. Además, la inhibición de la cadena respiratoria resulta en mayor producción de especies reactivas de oxígeno [37] y en la depleción de ATP en la célula y por ende en una incapacidad de llevar a cabo los procesos dependientes de él, entre ellos la degradación a través del proteosoma [37].

Este mecanismo se retroalimenta positivamente, puesto que se ha observado que el proteosoma es responsable de degradar proteínas mitocondriales mal plegadas, ya sean de la membrana externa, membrana interna o la matriz, lo cual es necesario para mantener funcional a esta organela [44,45]. Al estar inhabilitado el sistema ubiquitina-proteosoma se da la acumulación de proteínas dentro de la mitocondria, lo cual la lleva a su muerte. La misma $\alpha$-sinucleína, la cual estará aumentada al estar bloqueado su sistema de eliminación, puede inhibir el complejo I, además de bloquear el proceso de reparación mitocondrial $[44,47]$. No debe ser de extrañar que el estrés oxidativo también pueda ocasionar degeneración mitocondrial [9]. Esto porque la mitocondria, al ser la entidad formadora de las especies reactivas, se encuentra altamente expuesta a ellas. Esta exposición a ellas facilita la agregación de proteínas mal plegadas, desencadenando el desmantelamiento de los complejos proteicos y la pérdida de integridad mitocondrial [44].

El estrés oxidativo ocurre cuando la producción de especies reactivas excede la capacidad de un sistema de neutralizarlas y/o eliminarlas [47] y es crítico para la muerte neuronal, y se considera característico de las enfermedades neurodegenerativas $[47,48]$. Nuevamente son de gran importancia las exploraciones post-mortem en tejido cerebral para demostrar esta afirmación. En estos cerebros se encuentran carbohidratos, lípidos y ácidos nucleicos oxidados, niveles elevados de marcadores de peroxidación lipídica como el malondialdehído, lo cual junto al hallazgo de proteínas nitradas en los cuerpos de Lewy nos habla de la existencia un estrés oxidativo en estos pacientes [37, 47].

La explicación de esta vulnerabilidad selectiva irónicamente parece residir en la dopamina misma, aunque esta no es neurotóxica, en su proceso de catabolismo se producen especies como quinonas, semiquinonas, superóxido y peróxido de hidrógeno [9]. La $\alpha$-sinucleína además favorece la formación de cúmulos de dopamina, los cuales almacenan hierro, dejándolo disponible para la formación del radical hidroxilo en la reacción de Fenton [47]. El envejecimiento favorece aún más la disponibilidad del hierro en este microambiente con la acumulación neuronal de neuromelanina [9].

La caída en los niveles de ATP ya explicada aumentará la liberación de glutamato, por ende sobreactivando el receptor NMDA. La entrada de calcio al interior de la célula consecuentemente aumenta, activa la sintasa de óxido nítrico y el óxido nitro resultante reacciona con el superóxido 
disponible, formando el altamente tóxico y reactivo peroxinitrito (causante de las proteínas nitradas en los cuerpos de Lewy) $[49,50]$.

La acumulación intramitocondrial de proteínas mal plegadas y la resultante muerte mitocondrial elevará a su vez el estrés oxidativo [44]. El efecto ya explicado de la disfunción del sistema ubiquitina-proteosoma como efector de esta acumulación le sitúa como un causante más "corriente arriba" de este estrés [45]. Como se puede apreciar, el estrés oxidativo es propiciado por las mismas vías que las especies reactivas del oxígeno favorecen, reforzando la idea de que todos estos mecanismos están fuertemente entrecruzados.

Otra fuente de estrés oxidativo es la microglia, componentes del sistema inmune innato del sistema central [9]. La microglia está silenciada normalmente, pero al ser activada, y en el Parkinson se encuentra sobreactivada, favorece la liberación de especies reactivas, mayoritariamente por acción de la NADPH oxidasa [9, 51]. Esta célula glial puede ser activada al censar proteínas desplegadas, neuronas muriendo o por la presencia de patógenos [51].

\section{USO DE PESTICIDAS EN AGROINDUSTRIA Y POSIBLE CONTAMINACIÓN DE ALIMENTOS}

Los pesticidas de uso común en agricultura, horticultura y uso doméstico producen cuadros de intoxicación que se observan en fumigadores, cosechadores, en consumidores de alimentos contaminados y por medio de ingestión accidental o intencional [52]. Sin embargo, esto no quiere decir que los pesticidas organoclorados no provoquen contaminación alimentaria, ya que de hecho existen varias vías de contaminación, desde tratamientos fitosanitarios y terapéuticos del ganado, hasta contaminación ambiental [27].

El DDT, primer pesticida organoclorado, ingresa al organismo por medio de la ingestión de alimentos, especialmente aquellos con un alto contenido de grasa [53]. A pesar de las restricciones en el uso de los pesticidas en diversos países a nivel mundial, su persistencia, producción y liberación al medio ambiente en todo el mundo asegura que la contaminación llegue al suministro mundial de alimentos [54].

Costa Rica es uno de los países que utilizan mayor cantidad de pesticidas a nivel mundial [55]; y esto repercute en la salud de los consumidores nacionales ya que, por ejemplo, entre 1980 y 1991 Estados Unidos detuvo más de medio millón de kilogramos de productos costarricenses debido a que contenían residuos de plaguicidas no permitidos o por encima de los límites de tolerancia. Los alimentos involucrados fueron: melón, piña, chayote, ayote, fresa, mora, culantro, coco, yuca y ñampí [56].

Es importante recalcar que en Costa Rica el DDT se usó continuamente durante aproximadamente tres décadas para el combate de la malaria y el control de plagas en las cosechas. En 1981 se restringió su uso y en 1998 se prohibió, pero los resultados de estudios que se realizaron alrededor de esos años muestran la presencia de este compuesto en el organismo de la persona [56]. Además, en un estudio realizado en 1997-1998 en el cantón de Pococí en Limón, se descubrió que los productos con mayor contenido de residuo de plaguicidas eran el chile dulce, la lechuga, el tomate, el repollo y el banano; y esos residuos pertenecían a plaguicidas organofosforados $\mathrm{y}$ organoclorados [56].

El Ing. Oscar Arias, quien fue consultado para el desarrollo de este artículo, recalca que un mal manejo integrado de alguna plaga termina provocando contaminación ambiental y resistencia al pesticida utilizado; lo que provoca la creación de nuevos pesticidas, con diferentes mecanismos de acción y toxicidad para lograr el control de la plaga, aumentando el peligro de mayor contaminación y efectos adversos, en el ambiente, que aún no han sido descubiertos.

Diferentes organizaciones, como la FDA (Agencia de Alimentos y Medicamentos de Estados Unidos) y el EPA (Agencia de Protección Ambiental 
de Estados Unidos), han establecido umbrales para estos químicos, el problema radica en que en una dieta se incluyen alimentos de todo tipo en los cuales puede existir uno o más residuos de pesticidas y debido a que poseen mecanismos de acción similares, la combinación de ellos podría tener un efecto sinérgico en el cuerpo y provocar mayores y más graves efectos secundarios. La exposición a pesticidas no solamente se restringe a un aumento en el riesgo de padecer la enfermedad de Parkinson, sino que también se ha asociado con otras enfermedades como cáncer, diabetes y otras [54].

Diversos estudios realizados demuestran la relación existente entre la exposición a pesticidas y el aumento en la incidencia de la enfermedad de Parkinson, tal es el caso de las islas Faroe (archipiélago en el mar de noruega, bajo jurisdicción de Dinamarca donde se analizó la creciente prevalencia de esta enfermedad y se concluyó que, aunque no se supo la causa exacta del gran número de casos de Parkinson, los factores ambientales y hereditarios no podían ser excluidos como causas posibles [57]. La población de las islas Faroe posee una alta exposición a pesticidas organoclorados y otros contaminantes tóxicos debido a su dieta y además, posee una prevalencia de Parkinson del doble de lo esperado; un suceso similar se da con pueblos esquimales de Groenlandia, quienes también poseen un aumento en los casos de la enfermedad de Parkinson y están en constante exposición a pesticidas organoclorados ya que poseen una dieta similar a la de los habitantes de las islas Faroe [13].

\section{CONCLUSIÓN}

Las causas del surgimiento de la enfermedad de Parkinson son inciertas hasta el momento, si bien es cierto factores ambientales como la exposición a pesticidas ha tomado importancia recientemente ya que afectan el sistema nervioso de manera similar a como lo hace esta enfermedad aumentando los riesgos de padecerla. Costa Rica definitivamente no es un país exento al riesgo de padecer enfermedades degenerativas de este tipo debido al uso excesivo e irracional de pesticidas como el DDT en el país. La exposición a los mismos, ya sea debido a razones laborales o a través de la dieta, constituye un factor que incrementa el riesgo para padecer de Parkinson esporádico.

\section{REFERENCIAS}

1. Dawson, T., Dawson, V. Rare genetic mutations shed light on the pathogenesis of Parkinson disease. J.Clin. Invest January 2003; 111(2):145-151.

2. Blesa, J., Phani, S., Jackson-Lewis, V., Przedborski, S. Classic and New Animal Models of Parkinson's Disease. J Biomed Biotechnol March 2012; 2012: 110.

3. Lewis, K., Su, Y., Jou, O., Ritchie, C., Foong, C., Hynan, L., et al. Abnormal Neurites Containing C-Terminally Truncated $\alpha$-Synuclein are present in Alzheimer's Disease without Conventional Lewy Body Pathology. Am J Pathol December 2010; 177(6): 3037-3050.

4. Goedert, M., Spillantini, M., Del Tredici, K., Braak, H. 100 years of Lewy Pathology. Nat.Rev.Neurol January 2013; 9:13-24.

5. Snell, R. Núcleos de la Base (Ganglios Basales) y sus Conexiones. En Neuroanatomía Clínica. Snell, R (ed.) Editorial Médica Panamericana. Buenos Aires, Argentina. 5ta edición 2003, pp316-321.

6. Lyons, M., Boucher, O., Patel, N., Birch, B., Evidente, V. Long-Term benefit of bilateral subthalamic deep brain stimulation on Camptocormia in Parkinson's Disease. Turkish Neurosurgery 2012; 22 (4): 489492.

7. Mazzoni, P., Shabbott, B., Cortés, J. Motor Control Abnormalities in Parkinson's Disease. Cold Spring Harb Perspect Med 2012 June; 2(6): 1-17.

8. Fernandez, H. Nonmotor complications of Parkinson disease. Cleveland Clinic Journal of Medicine July 2012; 79(2): 14-18.

9. Varçin, M., Bentea, E., Michotte, Y., Sarre, S. Oxidative Stress in Genetic Mouse Models of Parkinson's Disease. Oxid Med Cell Longev July 2012; 2012: 1-25.

10. Horowitz, MP., Greenamyre, JT. Gene-Environment Interactions in Parkinson's Disease: The Importance

Revista electrónica publicada por el Departamento de Farmacología de la Escuela de Medicina de la Universidad de Costa Rica, 2060 San José, Costa Rica. ${ }^{\circledR}$ All rights reserved. Licensed under a Creative Commons Unported License. 
of Animal Modeling. Clin Parmacol Ther October $2010 ; 88(4): 467-474$.

11. Tanner, C., Ottman, R., Goldman, S., Ellenberg, J., Chan, P., Mayeux, R., et al. Parkinson Disease in Twins. An Etiologic Study. American Medical Association January 1999; 281 (4): 341-346.

12. Menegon, A., Board, P., Blackburn, A., Mellick, G., Le Couteur, D. Parkinson's disease, pesticides and glutathione transferase polymorphisms. The Lancet October 1998; 352: 1344-1346.

13. Richardson, J, Shalat, S., Buckley, B., Winnik, B., O'Suilleabhain, P., Diaz-Arrastia, R., et al. Elevated Serum Pesticide Levels and Risk of Parkinson Disease. Arch Neurol 2009 July; 66(7): 870-875.

14. Van der Mark, M., Brouwer, M., Kromhout H., Nijssen, P., Huss, A., Vermeulen, R. Is Pesticide Use Related to Parkinson Disease? Some Clues to Heterogeneity in Study Results. Environmental Health Perspectives 2012 March; 120 (3): 340-347.

15. Dick, F., De Palma, G., Ahmadi, A., Scott, N., Prescott, G., Bennett, J., et al. Environmental risk factors for Parkinson's disease and parkinsonism: the Geoparkinson study. Occup Environ Med February 2007; 64: 666-672.

16. Fong, CS., Wu, RM., Shieh, JC., Chao, YT., Fu, YP., Kuao, CL., et al. Pesticide exposure on southwestern Taiwanese with MnSOD and NQ01 polymorphisms is associated with increased risk of Parkinson's disease. Clin Chim Acta 2007 March; 378 (1-2): 136141.

17. Badii, M., Varela, S. Insecticidas organofosforados: Efecto sobre la Salud y el Ambiente. CULCyT Toxicología de Insecticidas 2008 SeptiembreOctubre; (28): 5-17.

18. Hall, W. Modulation of Movement by the Basal ganglia. En Neuroscience. Augustine, G., Fitzpatrick, D., Hall, W., McNamara, J., Purves, D., Williams, M. (eds.)

Sinauer Associates, Inc. Massachussetts, United States. $3^{\text {rd }}$ edition 2004, pp417-433.

19. Wichmann, T., Dostrovsky, J. Pathological basal ganglia activity in movement disorders. Neuroscience 2011 December; 198: 232-244.

20. Crittenden, J., Graybiel, A. Basal Ganglia Disorders Associated with Imbalances in the Striatal Striosome and Matrix Compartments. Front Neuroanat July 2011; 5: 1-25.
21. Redgrave, P., Rodriguez, M., Smith, Y., RodriguezOroz, M., Lehericy, S., Bergman, H., et al. Goaldirected and habitual control in the basal ganglia: implications for Parkinson's disease. Nat Rev Neurosci 2010 November;11 (11): 760-772.

22. Martínez, M., Martínez, S., Puelles, L. Sistema de los Ganglios Basales. En Neuroanatomía. Martínez, M., Martínez, S., Puelles, L. (eds.)

Editorial Médica Panamericana. Madrid, España. Primera Edición 2008, pp50-53.

23. Davis, M., Puhl, H. Nr4a1-eGFP is a Marker of Striosome-Matrix Architecture, Development and Activity in the Extended Striatum. PLoS One 2011 January; 6(1): 1-17.

24. Schwartz, M., Sabetay, S. An approach to the continuous dopaminergic stimulation in Parkinson's Disease. IMAJ March 2012; 14: 175-179.

25. Toxopeus, C., Maurits, N., Valsan, G., Conway, B., Leenders, K., de Jong, B. Cerebral Activations Related to Ballistic, Stepwise Interrupted and Gradually Modulated Movements in Parkinson Patients. PLoS One July 2012; 7(7): 1-15.

26. Lewis, K., Su, Y., Jou, O., Ritchie, C., Foong, C., Hynan, L., et al. Abnormal Neurites containing C-Terminally Truncated $\alpha$-synuclein are present in Alzheimer's Disease without conventional Lewy Body pathology. Am J Pathol December 2010; 177(6): 3037-3050.

27. Hernandez Rodriguez M., Sastre Gallego A. La cadena alimentaria como riesgo para la salud pública. En: Tratado de Nutrición. Ediciones Díaz de Santos. Madrid, España. Primera Edición 1999, pp 524-525.

28. RAP-Chile Alianza para una mejor calidad de vida. Alternativas al lindano, para el control de piojos y sarna en humanos y animales y la protección de semillas.http://www.rap-

al.org/articulos files/Lindano\%20Folleto.pdf. 2006. Accesada el 22 de Abril de 2013.

29. BAYER. Mecanismos de acción de los ectoparasiticidas.

http://mse.bayersanidadanimal.com.mx/ipublish/d ata/files/Familias Ectoparasiticidas y

Mecanismos Accion.pdf. S.f. Accesada el 7 de Febrero de 2013.

30. Routt, J. ,Roberts, J. Insecticidas Organofosfatados. En: Reconocimiento y Manejo de los Envenenamientos por Pesticidas. Lloyd, J., Keifer, M., 
Snodgrass, W., Wagner, S. (eds). Editorial United Book Press. Baltimore, Estados Unidos. Quinta Edición 1999, pp 40-54.

31. Pino, A., Brezmes, M. Intoxicación por organofosforados. $\quad$ www.secip.com/.../204intoxicacion-por-organofosforado... 2012. Accesada el 4 de Febrero de 2013.

32. Schafer, K., Reeves, M., Spitzer, S., Kegley, S. Chemical Trespass: Pesticides in our body and Corporate Accountability.

http://www.panna.org/sites/default/files/ChemTre sMain(screen).pdf. 2004. Accesada el 11 de Febrero de 2013.

33. García, C. Parkinson y pesticidas. http://repositorio.ual.es/jspui/bitstream/10835/10 95/1/garcia \%20martinez.pdf. 2012. Accesada el 7 de Febrero de 2013.

34. Borland, M., Trimmer, P., Rubinstein, J., Keeney, P., Mohanakumar, KP., Liu, L., et al. Chronic, low-dose rotenone reproduces Lewy neuritis found in early stages of Parkinson's disease, reduces mitochondrial movement and slowly kills differentiated SH-SY5Y neural cells. Mol Neurodegener December 2008; 3(21): 1-12.

35. Hughes, V. Misfolded protein transmits Parkinson's from cell to cell. https://sibdi.ucr.ac.cr/http://www.nature.com/new s/misfolded-protein-transmits-parkinson-s-fromcell-to-cell-1.11838 Accesada el 5 de Febrero, 2013.

36. Mishizen-Eberz, A., Guttman, R., Giasson, B., Day III, G., Hodara, R., Ischiropoulos, H., et al. Distinct cleavage patterns of normal and pathologic forms of $\alpha$-synuclein by calpain I in vitro. Journal of Neurochemistry August 2003; 86(4): 836-847.

37. Gandhi, S., Wood, N. Molecular pathogenesis of Parkinson's Disease. Hum. Mol. Genet September 2005; 14 (18): 2749-2755.

38. Sevlever, D., Jiang, P., Yen, SH. Cathepsin D is the main lysosomal enzyme involved in the degradation of $\alpha$-synuclein and generation of its carboxyterminally truncated species. Biochemistry September 2008; 47(36): 9678-9687

39. Lewis, K., Yaeger, A., DeMartino, G., Thomas, P. Accelerated Formation of $\alpha$-synuclein oligomers by concerted action of the $20 \mathrm{~S}$ proteasome and familial Parkinson mutations. J Bioenerg Biomembr February 2010; 42(1): 85-95
40. Fishman-Jacob, T., Reznichenko, L., Youdim, M., Mandel, S. A Sporadic Parkinson disease Model via silencing of the Ubiquitin Proteasome/E3 Component SKP1A. J Biol Chem November 2009; 284 (47): 32835-32845.

41. Eriksen, J., Wszolek, Z., Petrucelli, L. Molecular Pathogenesis of Parkinson Disease. Arch Neurol March 2005; 62 (3): 353-357.

42. Haass, C., Kahle, P. Neurodegenerative disease: Parkinsin's pathology in a fly. https://sibdi.ucr.ac.cr/http://www.nature.com/natu re/journal/v404/n6776/full/404341a0.html Accesada el 5 de Febrero, 2013

43. Kim, Y., Jang, W., Quezado, M., Oh, Y., Chul, K., Junn, E., et al. Proteasome Inhibition induces $\alpha$-synuclein SUMOylation and aggregate formation. J Neurol Sci August 2011; 307 (1-2): 157-161

44. Heo, J., Rutter, J. Ubiquitin-dependent mitocondrial protein degradation. Int $\mathrm{J}$ Biochem Biol October 2011; 43(10): 1422-1426

45. Henchcliffe, C., Flint, M. Mitochondrial biology and oxidative stress in Parkinson disease pathogenesis. Nature Clinical Practice Neurology November 2008; 4(11): 600-609.

46. Yoshii, S., Kishi, C., Ishihara, N., Mizushima, N. Parking mediates Proteasome-dependent protein degradation and ruptura of the Outer Mitochondrial Membrane. J Biol Chem June 2011; 286(22): 1936019640.

47. Pimentel, C., Batista-Nascimento, L., RodriguesPusada, C., Menezes, R. Oxidative stress in Alzheimer's and Parkinson's Diseases: Insights from the Yeast Sacchorymices cerevisiae. Oxid Med Cell Longev June 2012; 2012: 1-9

48. Roy, A., Ghosh, A., Jana, A., Liu, X., Brahmachari, S., Gendelman, H., et al. Sodium Phenylbutyrate Controls neuroinflammatory and antioxidant activities and protects dopaminergic neurons in mouse models of Parkinson's disease. PLoS One June 2012; 7(6): 1-18.

49. Lee, J., Giordano, S., Zhang, J. Autophagy, mitocondria and oxidative stress: cross-talk and redox signaling. Biochem J January 2012; 441: 523-540.

50. Perfeito, R., Cunha-Oliveira, T., Rego, A. Revisiting oxidative stress and mitocondrial dysfunction in the

Revista electrónica publicada por el Departamento de Farmacología de la Escuela de Medicina de la Universidad de Costa Rica, 2060 San José, Costa Rica. ${ }^{\circledR}$ All rights reserved. Licensed under a Creative Commons Unported License. 
pathogenesis of Parkinson disease - resemblance to the effect of amphetamine drugs of abuse. Free radical Biology and Medicine November 2012; 53(9): 1791-1806.

51. Peterson, L., Flood, M. Oxidative Stress and Microglial Cells in Parkinson's Disease. Mediatos Inflamm March 2012; 2012:1-12.

52. Cabrera, A., Varela, W. Intoxicación por Organofosforados (Revisión del Diagnóstico y Manejo). Revista Médica de Costa Rica y Centroamérica 2009; LXVI (588): 161-167.

53. Fernícola, N. Toxicología de los insecticidas organoclorados. Boletín de la Oficina Sanitaria Panamericana 1985 Enero; 98 (1): 10-19.

54. Schafer, K., Kegley, S. Persistent toxic chemicals in the US food supply. J Epidemiol Community Health 2002;56:813-817.

55. De los Santos, J., Pratt, L., Pérez, J. Uso de plaguicidas en la Agroindustria de Costa Rica. http://www.incae.edu/ES/clacds/publicaciones/pdf/ce n708.pdf. 1997. Accesada el 16 de Abril de 2013.

56. Organización Panamericana de la Salud. Efectos de los plaguicidas en la salud y el ambiente en Costa Rica.

http://www.bvs.sa.cr/php/situacion/plaguicidas.pdf. 2003. Accesada el 16 de Abril de 2013.

57. Wermuth, L., Joensen, P., Bunger, N., Jeune, B. High prevalence of Parkinson's disease in the Faroe Islands. Neurology 1997 March;49:426-432.

\section{Acerca de los Autores}

1- Sequeira Quesada, Carlos Mario carlossq24@hotmail.com Estudiante de medicina en el Hospital México. Uruca, Costa Rica.

2- Arias Rivera, Daniela nela2193@hotmail.com Estudiante Escuela de Nutrición de la Universidad de Costa Rica. San Pedro, Costa Rica. 